\title{
Intake of different types of red meat, poultry, and fish and incident colorectal cancer in women and men: results from the Malmö Diet and Cancer Study
}

\author{
Alexandra Vulcan ${ }^{\mathrm{a}}$, Jonas Manjer (10 ${ }^{\mathrm{b}}$, Ulrika Ericson (10) ${ }^{\mathrm{c}}$ and Bodil Ohlsson (1) ${ }^{\mathrm{a}}$ \\ aDivision of Internal Medicine, Department of Clinical Science, Lund University, Skåne University Hospital, Malmö, Sweden; ${ }^{\mathrm{b} D i v i s i o n}$ of \\ Surgery, Department of Clinical Science, Lund University, Skåne University Hospital, Malmö, Sweden; 'Diabetes and Cardiovascular Disease, \\ Genetic Epidemiology, Lund University, Skåne University Hospital, Malmö, Sweden
}

\begin{abstract}
Background: Colorectal cancer (CRC) is considered one of the most common forms of cancer in the Western world. High intake of red and processed meat is considered to increase CRC development.

Objective: This study examined associations between intake of red meats, poultry, and fish and incident $\mathrm{CRC}$, and if weight status modifies the associations.

Design: In the Malmö Diet and Cancer Study, dietary data was collected through a modified diet history method. Via the Swedish Cancer Registry, 728 cases of CRC were identified during 428924 person-years of follow-up of 16944 women and 10987 men.

Results: Beef intake was inversely associated with colon cancer. However, in men high intake of beef was associated with increased risk of rectal cancer. High intake of pork was associated with increased incidence of CRC, and colon cancer. Processed meat was associated with increased risk of CRC in men. Fish intake was inversely associated with risk of rectal cancer. No significant interactions were found between different types of meat and weight status.

Conclusions: Findings suggest that associations between meat intake and CRC differ depending on meat type, sex, and tumor location in the bowel. Weight status did not modify observed associations.
\end{abstract}

\section{ARTICLE HISTORY}

Received 22 December 2016 Accepted 31 May 2017

\section{KEYWORDS}

BMI; colorectal cancer; colon cancer; rectal cancer; sex; red meat

\section{Introduction}

Colorectal cancer (CRC) is estimated to be one of the most common forms of cancer in the Western world $[1,2]$. In Sweden, it is the fourth most common form of cancer and around 6500 persons per year develop the disease [3].

Several lifestyle factors are considered to be associated with the development of CRC. Higher body mass index (BMI) and waist circumference are positively associated with risk of CRC, independent of location, sex. or geographic area, as seen in a metaanalysis by $\mathrm{Ma}$ et al. [4]. Other lifestyle factors, such as diet, are also considered to be associated with risk of CRC. The World Cancer Research Fund and the American Institute for Cancer Research (WCRF/ AICR) have concluded that there is convincing evidence that high intakes of red and processed meat are associated with increased risk of CRC [5]. A metaanalysis indicated that the risk increases by $17 \%$ per $100 \mathrm{~g}$ daily intake of red meat and by $18 \%$ per $50 \mathrm{~g}$ daily intake of processed red meat [6]. According to the guidelines from the National Food Agency in Sweden, the intake of red meat should not exceed $500 \mathrm{~g}$ per week [7].

The risk associations may differ between gender and tumor location in the bowel [8]. In a meta-analysis by Alexander et al. [9], the association between high intake of processed meat and CRC was mainly seen in men. Although rather few studies have examined specific types of red meat in relation to colorectal cancer, a recent meta-analysis (2015) indicate that the associations differ depending on meat subtypes and sub-sites of CRC. High intakes of beef associated with risk of colon cancer, but not with rectal cancer. Lamb intake was suggested to associate with increased risk of CRC. Intake of poultry was not associated with CRC, but the authors concluded that more studies on pork intake are warranted [10]. Findings regarding high fish intake indicate an inverse association with risk of CRC [11]. 
The primary objective of the present study was to examine if intake of red meat, considering different subgroups, such as beef and pork, unprocessed and processed red meat, fish, and poultry, is associated with incident CRC, colon cancer, and rectal cancer in women and men from the Malmö Diet and Cancer Study (MDCS). Since obesity may promote development of CRC, our second objective was to investigate whether weight status may modify the association between meat intakes and CRC.

\section{Subjects and methods}

The study was approved by the Regional Ethical Review Board in Lund (50-81, 2013/804).

\section{Subjects}

MDCS is a population-based prospective cohort study in Malmö, Sweden. All men and women living in Malmö between 1991 and 1996, born between 1923 and 1950, were invited to participate. Altogether, 28098 participants completed all of the baseline examinations after having given their written informed consent. Of those having completed the baseline examination, 167 had been diagnosed with CRC before or at baseline examination, and were therefore excluded from the present study.

\section{Data collection}

At baseline, the participants were asked to fill out questionnaires on socioeconomic, lifestyle, and dietary factors. They also recorded their cooked meals and underwent a diet history interview. Weight, height, and waist circumference were measured by trained nurses. Body composition was estimated with a single-frequency bio-impedance methodology (BIA 103, RJL systems, Detroit, USA). Body fat percentage was calculated using an algorithm provided by the manufacturer.

\section{Dietary data}

Dietary data was gathered through a modified diet history method with a seven-day menu book for registration of meals that varied from day to day, most likely lunch and dinner, cold beverages, and nutrient supplements. In addition, the participants were given a 168-item questionnaire for assessment of consumption frequencies and portion sizes of foods that were not covered in the menu book. Finally, a 45-minute interview completed the dietary assessment. The merged data from the above mentioned methods was then coded using the Swedish Food Data Base [12]. The MDC diet assessment method has been described in detail elsewhere $[13,14]$.

The diet analyses were adjusted for the variables called 'method version' and 'season'. Method version was used because altered coding routines of dietary data were introduced in September 1994 in order to shorten the interview time (from one hour to 45 minutes). This resulted in two slightly different method versions, before and after September 1994, but did not have any major influence on ranking of individuals [13]. The variable season was divided into spring, summer, autumn, and winter depending on when in the year the baseline examination was executed. Dietary change in the past (yes, no) was based on the question 'Have you substantially changed your eating habits because of illness or some other reasons?' The relative validity of the MDCS method was evaluated in the Malmö Food study 1984-1985 in a sample of Malmö residents, 105 women and 101 men, 50-69 years old. An 18-day weighted food record was used as the reference method, three days every second month during a year $[15,16]$. The Pearson correlation coefficients, adjusted for total energy, between the reference method and the MDCS method were 0.70/0.74 (carbohydrates), $0.69 / 0.64$ (fats), $0.53 / 0.54$ (proteins), $0.69 / 0.74$ (fibers), $0.70 / 0.35$ (fish), $0.51 / 0.43$ (low fat meat) and 0.80/0.40 (high fat meat), in women and men, respectively.

The following variables for nutrient intake were used in this study: total energy (MJ), non-alcoholic energy (MJ), carbohydrates (percentage of energy (E $\%)$ ), fat (E\%), protein (E\%), saturated fat (g), fiber (g), calcium $(\mathrm{mg})$, folate $(\mathrm{mg})$, iron $(\mathrm{mg})$, and zinc $(\mathrm{mg})$. The following daily intakes of foods were used in this study: red meat $(\mathrm{g})$, unprocessed red meat $(\mathrm{g})$ processed red meat (sausages and cured meat) (g), beef $(\mathrm{g})$, pork $(\mathrm{g})$, poultry $(\mathrm{g})$, fish $(\mathrm{g})$, dairy products (portions of milk, yogurt sour milk, and cheese), fruit and berries (g), and sugar-sweetened beverages (g). Red meat was defined as pork, beef and game. Total red meat included both processed and unprocessed red meat. Intakes of pork and beef were mainly based on non-processed meat, as distinction between pork and beef was not possible for all processed meats based on items included in the food questionnaire. The fish variable consisted of both processed and unprocessed fish. Portions, instead of grams, were used in order to analyse the sum of dairy products, because of different water content and because they usually are consumed in different weights. Standard portion sizes from the national Food Agency in Sweden were used to define portions for dairy products [17].

Energy-adjusted variables were obtained by regressing the food intakes on non-alcohol energy intake 
[18]. Quintiles of the food residuals were used as exposure categories.

\section{Cancer cases}

In the study, 728 cases of CRC were identified from the Swedish Cancer Registry, of which 463 were colon cancer and 265 rectal cancers, during 428924 person-years of follow-up. Follow-up time was defined as the time from date of enrolment until date of CRC-diagnosis, death, migration, or end of follow-up (31 December 2010), whichever came first. Information on date of death was collected from The Swedish Cause-of-death registry. Mean follow-up was 15.4 years.

\section{Other variables}

Prevalent diabetes diagnosis was determined from selfreported diagnosis, self-reported medication for diabetes, or information from medical data registries indicating a date of diagnosis before inclusion in the MDCS. Incident diabetes diagnosis was obtained either from the Regional Diabetes 2000 register of Scania, the Malmö HbA1C register or the Swedish National Diabetes Register. In the MDCS, 3245 incident cases of diabetes and 1183 prevalent cases of diabetes were found. In all those with diabetes, 185 cases of incident CRC were found.

Age was obtained from personal identification numbers. Smokers were divided into three categories: current smokers; ex-smokers; and non-smokers. Irregular smoking was counted as current smoking. The subjects estimated their physical activity in minutes and the results were divided into quintiles. The level of education was divided into four different categories: $\leq 8$ years; $9-10$ years; $11-13$ years of education; and university degree. Alcohol intake was divided into four categories: zero; $<15 \mathrm{~g} / \mathrm{d}$ for women and $<20 \mathrm{~g} / \mathrm{d}$ for men; $15-30 \mathrm{~g} / \mathrm{d}$ for women and 20-40 g/ $\mathrm{d}$ for men; and $>30 \mathrm{~g} / \mathrm{d}$ for women and $>40 \mathrm{~g} / \mathrm{d}$ for men. Current use of menopausal hormonal replacement therapy (HRT) and regular use of non-steroid anti-inflammatory drugs (NSAID) were divided into non-users and users. BMI was calculated from measured weight and length. BMI was divided into high $\left(\geq 25 \mathrm{~kg} / \mathrm{m}^{2}\right)$ and normal and low $\left(<25 \mathrm{~kg} / \mathrm{m}^{2}\right)$, after the World Health Organization's classification of overweight [19].

\section{Statistical methods}

The SPSS statistics package (version 22; IBM Corporation, Armonk, USA) was used for all statistical analyses. The food variables were log-transformed (e-log) to normalize the distribution before analysis. A very small amount (0.0001) was added before transformation, to handle zero intakes [20].

The general linear model was used when examining baseline continuous characteristics in the cases and noncases, and also adjusting for age and sex, when appropriate, and season and method version for the food variables.

The Cox proportional hazard regression model was used when estimating hazard ratios (HR) of incident CRC, colon cancer, and rectal cancer, depending on quintiles of energy-adjusted food intakes (red meat, beef, pork, unprocessed red meat, processed red meat, poultry, and fish). The basic model included adjustments for age, sex (when appropriate), season, and total energy intake. In addition, the full model was also adjusted for level of education, smoking status, alcohol intake, physical activity, BMI, NSAID use, and when appropriate, for current use of HRT. We also performed the multivariate model excluding BMI, since it might be an intermediate between diet and CRC. Additional models were constructed with further adjustments for diabetes (prevalent and incident) or potential dietary confounders found in previous studies (intake of: fiber; protein; saturated fat; calcium; folate; iron; zinc; fruits and vegetables; milk products; and sugar-sweetened beverages) [5].

Spearman's correlation matrix was used to examine the correlation between energy-adjusted food intakes (total red meat, beef, pork, unprocessed red meat, processed red meat, poultry, and fish). For intakes where a correlation over 0.40 was found (Supplementary Table 1), additional models were constructed with mutual adjustment in the full models.

A test for interaction between sex or BMI status $(<25$ and $\geq 25)$ and dietary intakes with regard to CRC incidence was performed by adding a multiplicative variable (e.g. sex $\times$ diet quintile (treated as continuous variables)) to the full model.

In sensitivity analysis, we excluded individuals with a reported dietary change in the past, all forms of prevalent cancer except cervix cancer in situ or incident and prevalent diabetes. All tests were two-sided and statistical significance was assumed at $\mathrm{p}<0.05$.

\section{Results}

\section{Baseline characteristics}

Altogether, 16944 (60.7\%) women and 10987 men completed the baseline examinations, after exclusion of individuals with prevalent CRC. The cases of CRC were, compared with non-cases, older, had a larger waist circumference, and a higher BMI (Table 1). They also had a lower intake of calcium, zinc, beef, 
and dairy products and a higher intake of pork. Fewer cases had a high level of education, but more of them were highly physically active. Diabetes was more common among the cases, especially in men. In women, the use of HRT was higher among the non-cases.

\section{Dietary intake and colorectal cancer}

In the full multivariate model, beef intake was inversely associated with risk for CRC in women (HR: 0.65 for highest compared with lowest quintile; $95 \%$ CI: $0.45,0.95$; $\mathrm{p}$ for trend $=0.046$ ), but not in men, and a borderline interaction between sex and beef intake was seen $(\mathrm{p}=0.068)$ (Table 2$)$. High pork intake was associated with increased incidence for CRC (HR: 1.39 for highest compared with lowest quintile; 95\% CI: 1.09, 1.78; $\mathrm{p}$ for trend $=0.023$ ). The association was only significant in women (HR: 1.54 for highest compared with lowest quintile; $95 \%$ CI: $1.12,2.15$; $\mathrm{p}$ for trend $=0.003)$, but no significant interaction with sex was seen $(\mathrm{p}=0.157)$. The trend for intake of processed red meat was significantly associated with increased risk for CRC in men (HR: 1.23 for highest compared with lowest quintile; $95 \%$ CI: $0.87,1.73 ; \mathrm{p}$ for trend $=0.023$ ), but not in women, and a borderline interaction was seen $(\mathrm{p}=0.062)$.

\section{Dietary intake and colon cancer}

High intake of beef was inversely associated with risk of colon cancer (HR: 0.60 for highest compared with lowest quintile; $95 \% \mathrm{CI}$ : 0.44, 0.82; $\mathrm{p}$ for trend $=0.009$ ) (Table 3). The inverse association was also significant in women (HR: 0.60 for highest compared with lowest quintile; 95\% CI: 0.37, 0.96; $\mathrm{p}$ for trend $=0.049$ ) and similar tendencies were seen in men ( $\mathrm{p}$ for trend $=0.069)$. High intake of pork was associated with increased risk of colon cancer (HR: 1.41 for highest compared with lowest quintile; 95\% CI: 1.04, 1.90; p for trend $=0.021)$. The association was only significant in women (HR: 1.56 for highest compared with lowest quintile; 95\% CI: 1.12, 2.15; $\mathrm{p}$ for trend $=0.003$ ), but no significant interaction with sex was seen $(\mathrm{p}=0.146)$. We observed a borderline significant association between high intake of processed meat and increased risk of colon cancer in men (HR: 1.23 for highest compared with lowest quintile; 95\% CI: 0.80, 1.90; p for trend $=0.053)$, but no significant interaction with sex was seen $(p=0.127)$.

\section{Dietary intake and rectal cancer}

High intake of beef was associated with increased risk of rectal cancer in men (HR: 1.82 for highest compared with lowest quintile; 95\% CI: 1.02, 3.25, p for trend $=0.028$ ), but not in women (Table 4 ), and a significant interaction was seen between beef intake and sex $(\mathrm{p}=0.025)$. High intake of fish was inversely associated with risk of rectal cancer in all (HR: 0.59 for highest compared with lowest quintile; $95 \%$ CI: 0.38 , 0.92 , $\mathrm{p}$ for trend $=0.025$ ), and the association did not differ depending on sex ( $p$ for interaction $=0.597$ ).

\section{Complementary models}

In the analysis of CRC, colon and rectal cancer, additional adjustments for potential dietary confounders (intake of: fiber; protein; saturated fat; calcium; folate; iron; zinc; fruits and vegetables; milk products; and sugar-sweetened beverages) did not affect the results and were therefore excluded from further analysis (data not shown).

In the analysis of CRC, colon, and rectal cancer, additional mutual adjustments for the correlated food intakes (total red meat - beef; total red meat - pork; total red meat - unprocessed red meat; total red meat processed red meat; unprocessed red meat - beef; unprocessed red meat - pork), did not affect the results to any major extent (data not shown).

\section{Dietary intake, colorectal, colon, and rectal cancer, and body mass index}

Excluding BMI from the multivariate model gave virtually similar results (data not shown). Moreover, no significant interactions were found between the different types of meat intakes and BMI status $(<25$ or $\geq 25)$ on CRC (Supplementary Table 2).

\section{Sensitivity analyses}

\section{Exclusion of subjects with dietary change}

When excluding individuals reporting dietary change in the past, the association between intake of pork and CRC was only borderline significant (HR: 1.15 for highest compared with lowest quintile; 95\% CI: 0.86, 1.52; $\mathrm{p}$ for trend $=0.074$ ). Apart from that, we did not observe any major changes in any of the results.

\section{Exclusion of subjects with prevalent cancer}

When excluding individuals with any type of prevalent cancer at baseline, except for cervix cancer in situ, the inverse association between intake of beef and colon 


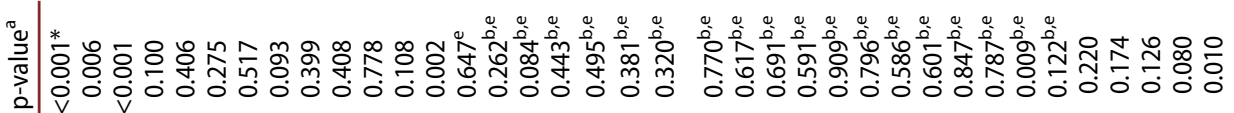

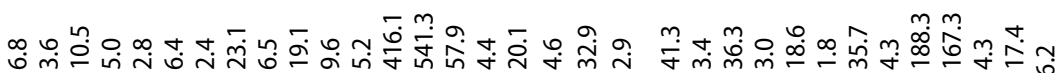

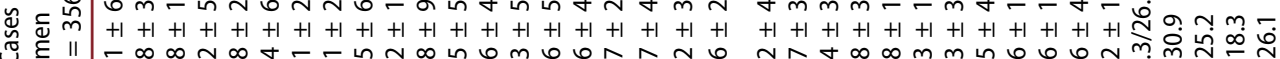

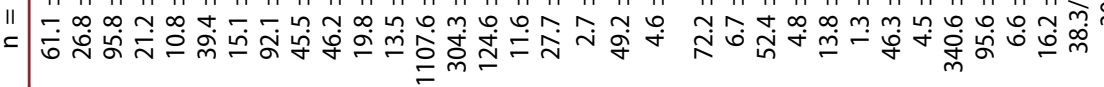

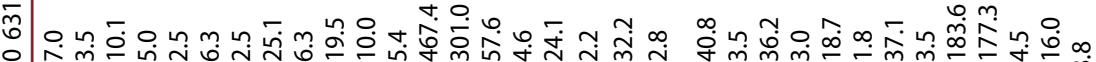

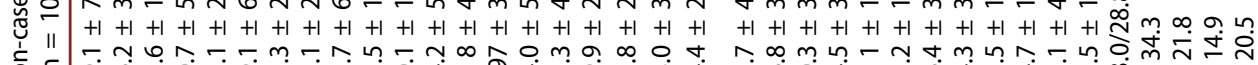

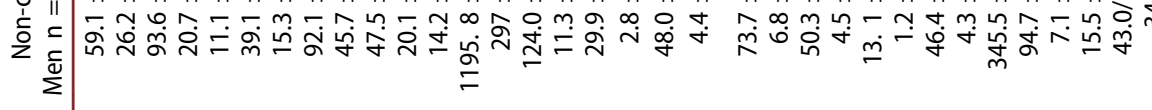

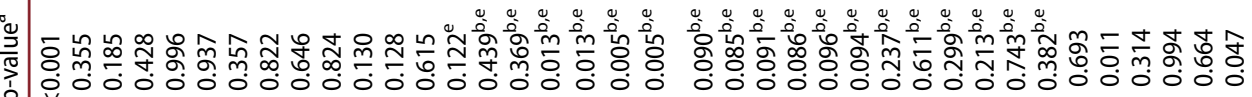

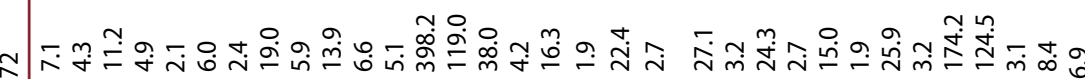

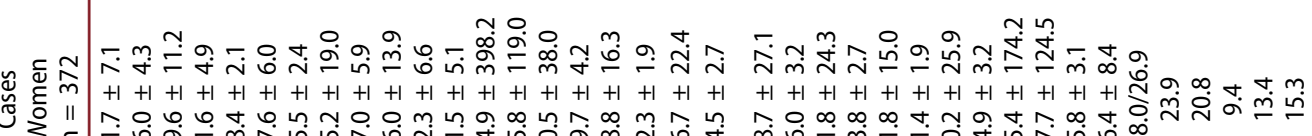

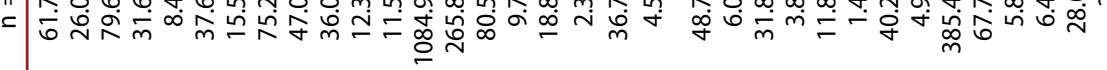

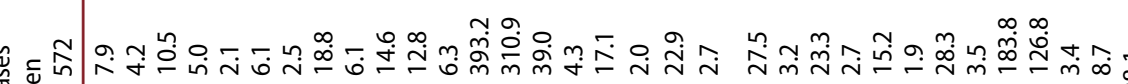

1
$\varepsilon$ 引 II

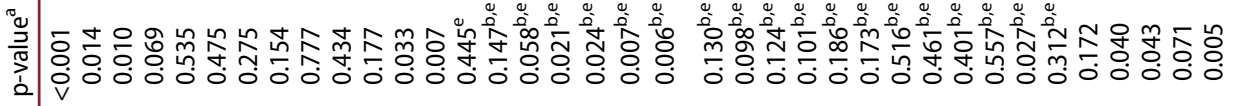

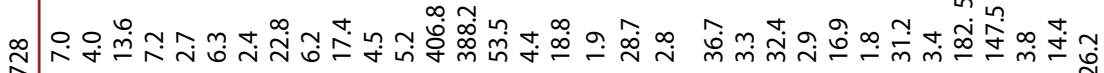

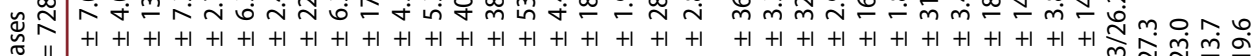

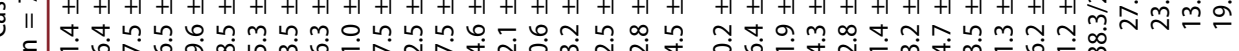

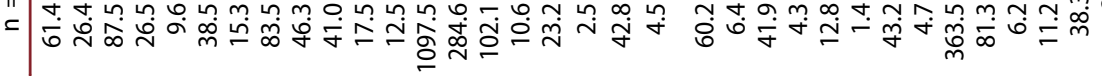

๕

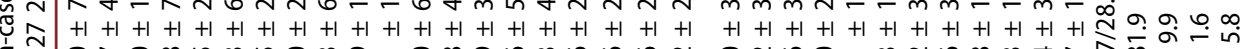

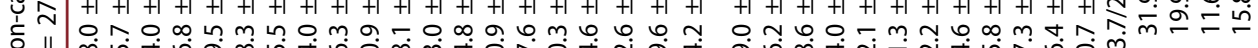

2 II 
$6 \Leftrightarrow$ A. VULCAN ET AL.

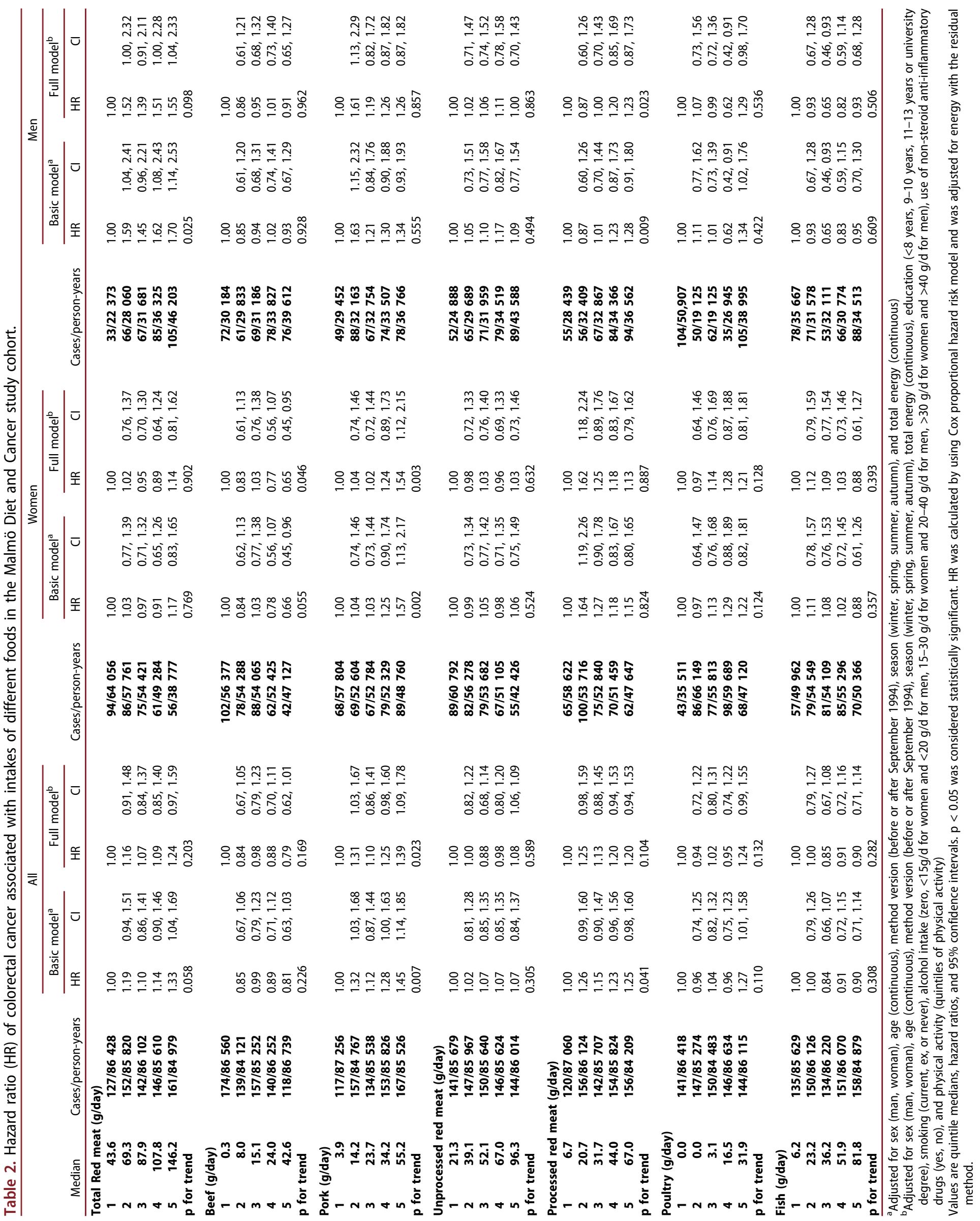




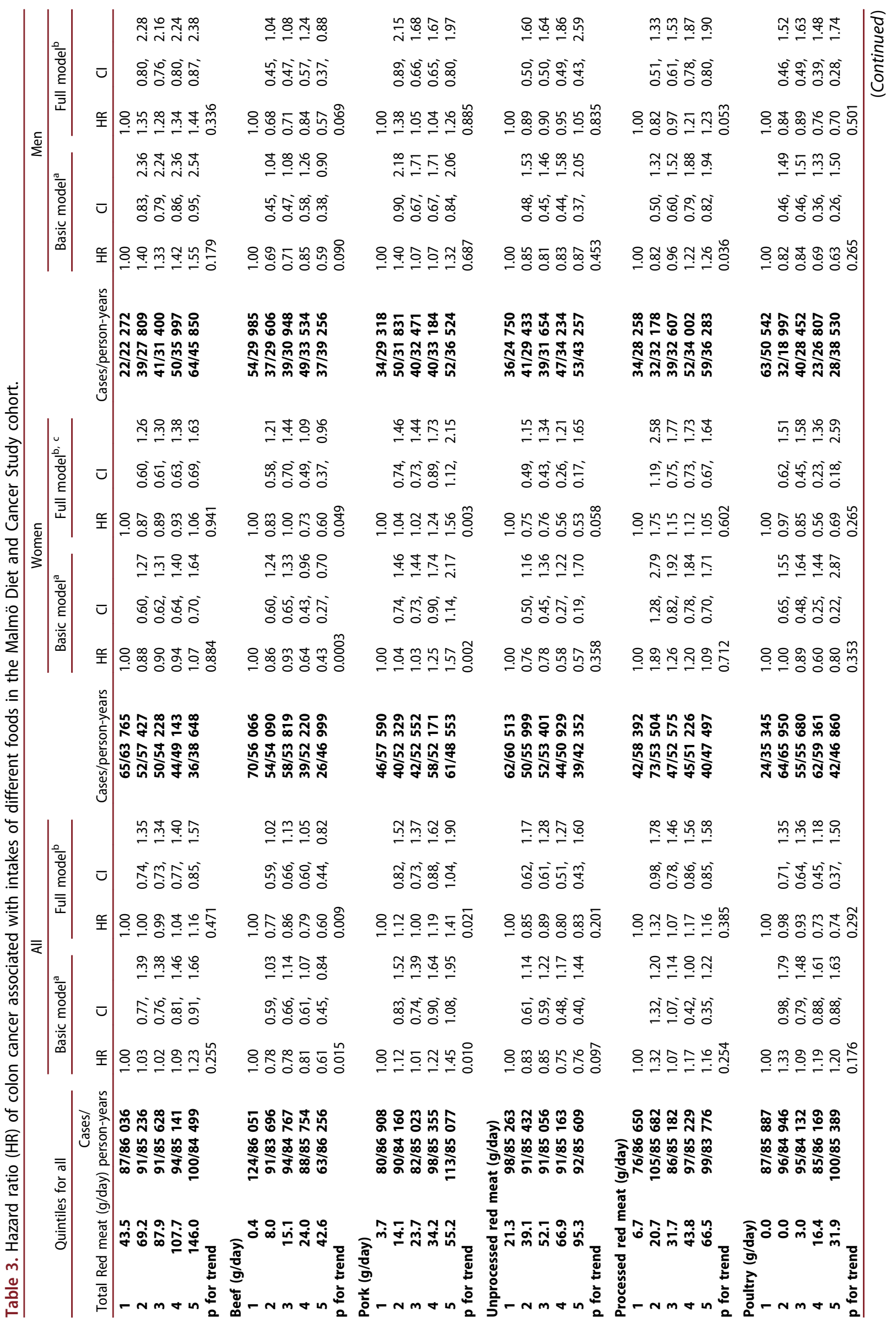




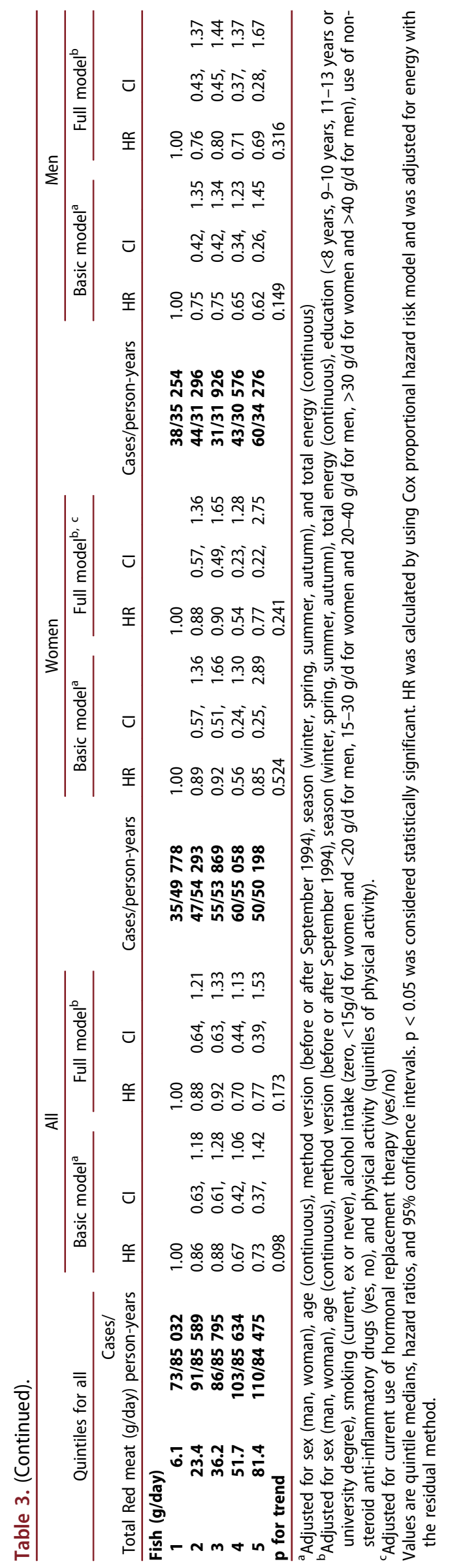




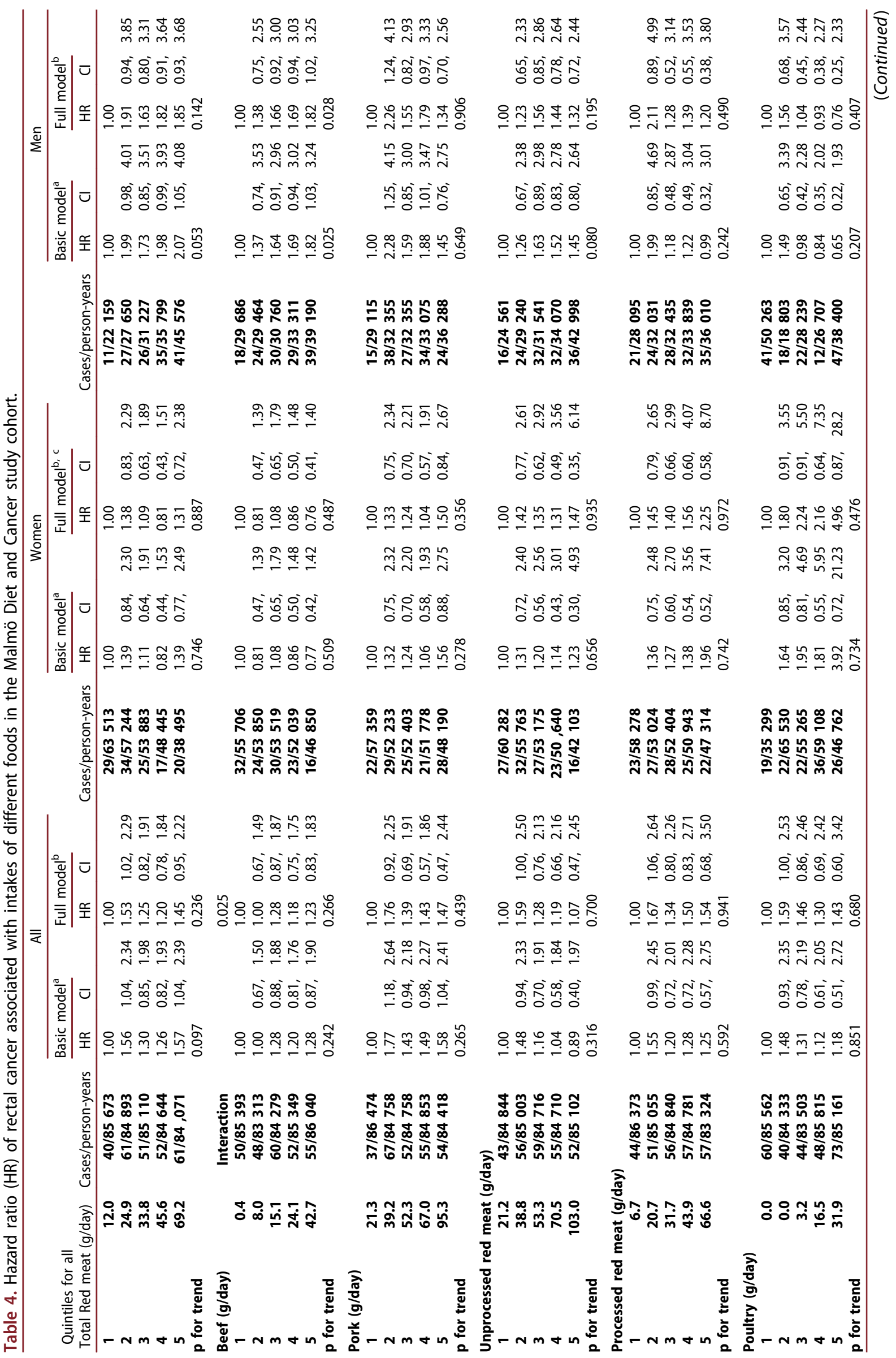




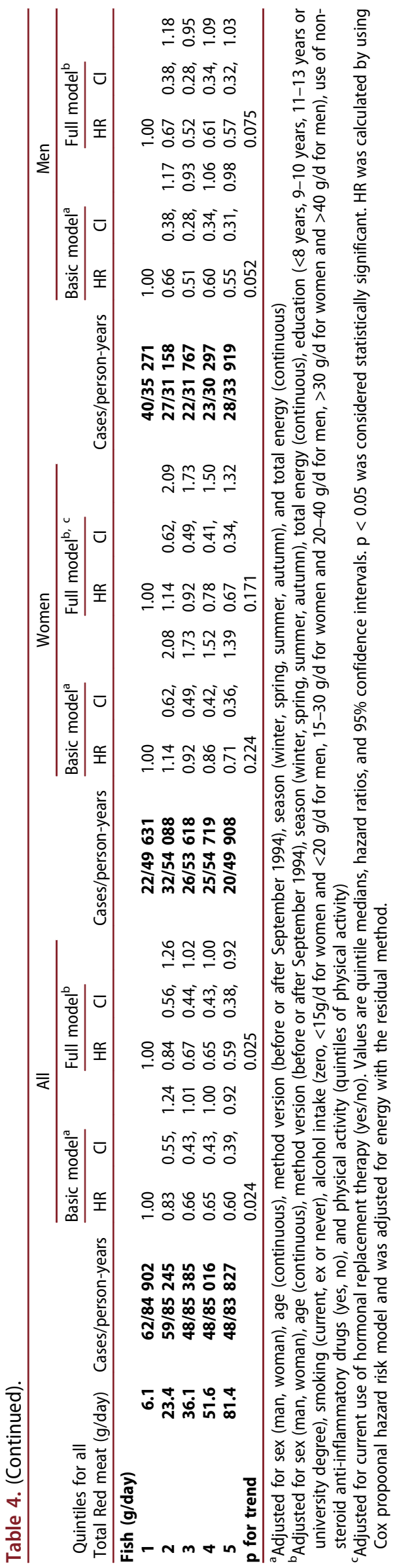

cancer became significant also in men (HR: 0.56 for highest compared with lowest quintile; $95 \%$ CI: 0.36 , 0.88; $\mathrm{p}$ for trend $=0.035$ ).

\section{Exclusion of subjects with prevalent or incident diabetes}

The association between high intake of processed red meat and risk of CRC in men, did not remain significant (HR: 1.05 for highest compared with lowest quintile; $95 \%$ CI: $0.71,1.56$; $\mathrm{p}$ for trend $=0.155$ ) when excluding individuals with diabetes.

\section{Discussion}

In the present study, high intake of pork was associated with increased risk of CRC, and especially with colon cancer in women. In contrast, high intake of beef was associated with decreased risk of colon cancer, whereas it was associated with increased risk of rectal cancer in men. Furthermore, there was a trend for increased risk of CRC with higher intake of processed meat among the men, mainly driven by colon cancer. Fish intake was inversely associated with rectal cancer.

In line with our findings, Bernstein et al. [8] did not observe high intakes of unprocessed red meat to be associated with a substantially increased risk of CRC when recent results from the Nurses' Health Study and the Health professionals Follow-up Study were pooled. Similarly to the meta-analysis by Alexander et al. [9], our results indicate a positive association between intake of processed red meat and CRC in men, but not in women, whereas other metaanalyses have not shown differing associations depending on sex [21,22]. Previous meta-analyses have also indicated significant associations between high intake of processed meat and colon cancer, but not with rectal cancer $[6,8]$, although Bernstein et al. [8] concluded that they could not find evidence to show that the associations differed with colon or rectal cancers, but that the intake of processed red meat was especially associated with increased risk of distal colon cancer. Few studies have examined subsites of colon malignancies, but comparable findings were seen in a meta-analysis of three earlier prospective studies [23], whereas positive associations with both proximal and distal colon cancer were seen in a Norwegian study [24]. In a recent meta-analysis (2015) comparing different meat subtypes, high intakes of beef and lamb, but not pork and poultry, were associated with increased risk of CRC [10]. However, when excluding one of the included cohorts due to heterogeneity between the studies, 
meta-analysis of four prospective studies indicated, similar to our results, an increased risk of CRC at high intakes of pork [10].

The diverse findings depending on type of meat, gender, and tumor site may reflect the complexity of colorectal cancer. Proximal colon and distal colon (including rectum) arise from different embryonic tissues. They also serve different functions, and the mucosal properties and microenvironment differ between segments [25]. As the fecal content is degraded by the microbiota and water and minerals are reabsorbed during the colonic passage, the production of short-chain fatty acids and metabolites varies, and fecal content changes its properties, in distal direction. Traditionally, we consider colon cancers as one disease. This may be misleading, since proximal colon cancer more often have microsatellite instability, CpG island methylator phenotype, and KRAS mutations, whereas rectal and distal colon cancers more often have chromosomal instability and TP53 and APC mutations [26]. So far, the incidence of distal cancer has been higher than the incidence of proximal cancer [27]. In future studies, subgroup analysis of proximal or distal CRC ought to be done in addition to analysis of colon and rectal cancer, which is more common in epidemiological studies and therefore more comparable.

Associations between meat intake and CRC may differ depending on the type of meat most frequently consumed in a different population, as well as on intake levels. In the southernmost district in Sweden, where participants of the MDCS cohort were enrolled, pork intake is by tradition high. Although the intake data on meat could be considered to be satisfactory, it is worth noting, that the main part of the meat intake was recorded in the MDCS sevenday menu book, and that fewer days are needed to capture intakes of food consumed more frequently, compared to those consumed more seldom, indicating that intake data on pork may be more valid, compared to that on beef. This may partly explain why beef was not found to associate with higher risk of colon cancer in this study, while such association has been observed in other populations $[9,10]$. On the other hand, difference in meat production, with fewer antibiotics and growth factors used in Sweden, compared with for example USA, may affect the association between beef intake and risk of CRC differently. It is stated that increased risk for CRC starts at an intake of $500 \mathrm{~g} /$ week for red meat and for processed meat only by eating it [5]. In the present study, the estimated median intake in the lowest (44 g/day), and the highest (146 g/day) quintile of red meat, was well below, and respectively above, the stated threshold level.
The gender difference in observed associations may have several explanations. The prolonged gastrointestinal transit time in women compared with men may lead to a prolonged exposure of the mucosa to carcinogens [28]. On the other hand, this may be counteracted by higher meat intake in men compared with that in women in the MDCS. If meat has carcinogen-inducing properties in the bowel, a difference in transit time may decrease the difference in risk between genders caused by difference in meat intake.

Several mechanisms may lie behind observed associations between red meats and CRC. One difference between beef and pork is the amount and composition of fat [29]. The generally higher fat concentration in pork may enhance the excretion of bile acids, which may promote tumorigenesis [30,31]. During the passage down the colon, the bile acids are modified and dehydroxylated [25], leading to different properties along the colon mucosa. Thus, the malignancy risk may vary between different colon segments. The heme iron in red meat is another plausible explanation for the association between red meats and CRC [29], as heme iron damages the colon's lining [32]. However, the inverse association between beef intake and risk of colon cancer in the present study, and the association between high pork intake and increased risk of CRC, do not support this explanation.

Consumption of red meat may result in exposure to carcinogens through cooking methods, such as cooking meat at a high temperature by barbequing or smoking, or through preservation with nitrite. Heterocyclic amines (HCAs), polycyclic aromatic hydrocarbons (PAHs), and N-nitroso compounds (NOCs) are thought to be factors in development of CRC $[33,34]$. In addition, processed meat often contains a high amount of salt [35] and it has been discussed if salt may be a risk factor for CRC [36].

Previous indications of protective associations with high fish intake have, consistent with our findings, above all been seen for rectal cancer [11]. Intake of marine omega- 3 is thought to be inversely associated with CRC [37]. Mechanisms are thought to be the reducing effect omega- 3 has on the omega- 6 polyunsaturated fatty acid production of eicosanoids, and inhibition of cyclo-oxygenase-2 [38], but when analyzing associations between omega- 3 intake and risk of CRC, no clear association was seen [5]. The high content of vitamin $\mathrm{D}$ and selenium has also been suggested as potential mechanisms [39]. This may explain the inverse association between fish and rectal cancer seen in the present study.

Obesity is considered to be an established risk factor for CRC [4]. Low grade inflammation and changes in 
microbiota are associated with obesity and have been discussed as possible causes [4], along with insulin-like growth factor-1 and leptin [40]. The observed associations in this study did not clearly differ between individuals with BMI above or below 25, and exclusion of BMI in the multivariate model did not substantially change our finding, suggesting that weight status does not have any modifying or mediating effects on the associations between meat intakes and CRC. Yet, we cannot exclude modification if further discriminating between overweight and obese in more well-powered studies.

Diabetes may promote development of CRC, and red and processed meats have in studies shown to be associated with increased risk of diabetes [41,42]. Exclusion of patients with diabetes did not significantly affect the association between intake of pork or beef and risk of CRC, but the association between intake of processed red meat and CRC did not remain significant in men and the risk estimate in the highest quintile changed from 1.23 to 1.05 . Loss of power may lie behind this observation; especially since diabetes is more common among men (26\% were excluded). Future well-powered studies may reveal if the presence of diabetes has any modifying effect.

The strength of the present study is dietary data of high relative validity $[13,15]$. As it is a large population-based prospective study with long follow-up, selection bias and reverse causation were minimized. We had also extensive information on potential confounding factors and were able to exclude individuals who reported dietary changes in the past. There are several limitations of this study. Power may be a problem in some of the gender specific analyses. Family history of CRC and inflammatory bowel disease, seen as strong risk factors in development of CRC [22], were not included in the study as information on these risk factors were missing. Furthermore, neither information on the source of some of the processed meat, which may have influenced the findings regarding intakes of beef and pork and their association with risk of CRC, nor information about intake of heme iron was available.

Despite adjustments for possible confounders and known risk factors, occurrence of residual confounding cannot be completely excluded and we have not adjusted for multiple testing, since dietary intakes are highly correlated and the analyses could not be treated as independent.

\section{Conclusion}

In conclusion, our findings suggest that type of meat as well as sex and tumor location in the bowel influence associations between meat intake and risk of CRC. The findings support previous studies indicating that high intake of processed meat above all is associated with increased risk of CRC in men and that high intake of pork may be associated with an increased risk of CRC. Beef intake was in contrast to previous observations inversely associated with colon cancer, but in men associated with increased risk of rectal cancer. Fish intake was inversely associated with rectal cancer. Presence of overweight did not seem to have any major impact on the findings.

\section{Acknowledgements}

This study was funded by grants from Development Foundation of Region Skåne, Direktör Albert Påhlsson's Foundation, and the Malmö Hospital Foundation for Cancer Prevention. The funders had no role in the design, analysis or writing of this article.

\section{Disclosure statement}

No potential conflict of interest was reported by the authors.

\section{ORCID}

Jonas Manjer (D) http://orcid.org/0000-0002-3158-5289

Ulrika Ericson (1) http://orcid.org/0000-0003-4629-4318

Bodil Ohlsson (D) http://orcid.org/0000-0002-9142-5244

\section{References}

[1] Ferlay J, Parkin DM, Steliarova-Foucher E. Estimates of cancer incidence and mortality in Europe in 2008. Eur J Cancer. 2010;46(4):765-781.

[2] Edwards BK, Ward E, Kohler BA, et al. Annual report to the nation on the status of cancer, 1975-2006, featuring colorectal cancer trends and impact of interventions (risk factors, screening, and treatment) to reduce future rates. Cancer. 2010;116(3):544-573.

[3] Bergman O, Hont G, Johansson E Cancer i siffror [Internet page]. Socialstyrelsen Cancerfonden; 2013 [cited 2013 september 18]. 20130605: Available from: http://www.socialstyrelsen.se/Lists/Artikelkatalog/ Attachments/19108/2013-6-5.pdf

[4] Ma Y, Yang Y, Wang F, et al. Obesity and risk of colorectal cancer: a systematic review of prospective studies. Plos One. 2013;8(1):e53916.

[5] World Cancer Research Fund and American Institute for Cancer Research. Continuous update project report. Food, Nutrition, Physical Activity, and the Prevention of Colorectal Cancer. 2011.

[6] Chan DSM, Lau R, Aune D, et al. Red and processed meat and colorectal cancer incidence: meta-analysis of prospective studies. Plos One. 2011;6(6):e20456.

[7] Bjerselius R, Konde ÅB, Färnstrand JS. Konsumtion av rött kött och charkuteriprodukter och samband med tjock- och ändtarmscancer av - risk- och nyttohanteringsrapport. Uppsala, Sweden: Livsmedelsverket; 2014. 
Available from: http://www.livsmedelsverket.se/globalas sets/matvanor-halsa-miljo/kostrad-matvanor/rott-kottoch-chark/livsmedelsverket.-konsumtion-av-rott-kottoch-charkuteriprodukter-och-samband-med-tjock-ochandtarmscancer.-rapport-20-2014.pdf?id=3573

[8] Bernstein AM, Song M, Zhang X, et al. Processed and unprocessed red meat and risk of colorectal cancer: analysis by tumor location and modification by time. Plos One. 2015;10(8):e0135959.

[9] Alexander DD, Miller AJ, Cushing CA, et al. Processed meat and colorectal cancer: a quantitative review of prospective epidemiologic studies. European Journal Cancer Prevention: Official Journal European Cancer Prevention Organisation. 2010;19(5):328-341.

[10] Carr PR, Walter V, Brenner H, et al. Meat subtypes and their association with colorectal cancer: systematic review and meta-analysis. Int J Cancer. 2015;138(2):293-302.

[11] Wu S, Feng B, Li K, et al. Fish consumption and colorectal cancer risk in humans: a systematic review and meta-analysis. Am J Med. 2012;125(6):551-9e5.

[12] Livsmedelsverk S. Livsedelstabeller. Stockholm: Liber tryck AB; 1986.

[13] Wirf?Lt E, Mattisson I, Johansson U, et al. A methodological report from the Malm? Diet and Cancer study: development and evaluation of altered routines in dietary data processing. Nutr J. 2002;1:3.

[14] Callmer E, Riboli E, Saracci R, et al. Dietary assessment methods evaluated in the Malmö food study. J Intern Med. 1993;233(1):53-57.

[15] Elmståhl S, Riboli E, Lindgärde F, et al. The Malmö Food Study: the relative validity of a modified diet history method and an extensive food frequency questionnaire for measuring food intake. Eur J Clin Nutr. 1996;50(3):143-151.

[16] Riboli E, Elmståhl S, Saracci R, et al. The Malmö Food Study: validity of two dietary assessment methods for measuring nutrient intake. Int J Epidemiol. 1997;26 (Suppl 1):S161-73.

[17] The Swedish National Food Adminstration (SLV). Vikttabell. Uppsala: The Swedish national Food Adminitration; 1999.

[18] Willett W, Stampfer MJ. Total energy intake: implications for epidemiologic analyses. Am J Epidemiol. 1986;124(1):17-27.

[19] WHO. BMI classification 2006. Updated 26 Novmeber 2015. Available from: http://apps.who.int/bmi/index. jsp?introPage=intro_3.html

[20] Margetts BM, Nelson M. Design Concepts in Nutritional Epidemiology:Norfolk:Oxford University Press, printed by Biddles LTd King's Lynn;1997.

[21] $\mathrm{Xu} \mathrm{X,} \mathrm{Yu} \mathrm{E,} \mathrm{Gao} \mathrm{X,} \mathrm{et} \mathrm{al.} \mathrm{Red} \mathrm{and} \mathrm{processed} \mathrm{meat} \mathrm{intake}$ and risk of colorectal adenomas: a meta-analysis of observational studies. Int J Cancer. 2013;132(2):437-448.

[22] Johnson CM, Wei C, Ensor JE, et al. Meta-analyses of colorectal cancer risk factors. Cancer Causes Control. 2013;24(6):1207-1222.

[23] Larsson SC, Wolk A. Meat consumption and risk of colorectal cancer: a meta-analysis of prospective studies. Int J Cancer. 2006;119(11):2657-2664.

[24] Parr CL, Hjartåker A, Lund E, et al. Meat intake, cooking methods and risk of proximal colon, distal colon and rectal cancer: the Norwegian Women and Cancer (NOWAC) cohort study. Int J Cancer. 2013;133(5):1153-1163.

[25] Iacopetta B. Are there two sides to colorectal cancer? Int J Cancer. 2002;101(5):403-408.

[26] Lee GH, Malietzis G, Askari A, et al. Is right-sided colon cancer different to left-sided colorectal cancer? - a systematic review. Eur J Surg Oncol. 2015;41(3):300-308.

[27] Beart RW, Melton LJ 3rd, Maruta M, et al. Trends in right and left-sided colon cancer. Dis Colon Rectum. 1983;26(6):393-398.

[28] Sadik R, Abrahamsson H, Stotzer PO. Gender differences in gut transit shown with a newly developed radiological procedure. Scand J Gastroenterol. 2003;38(1):36-42.

[29] Livsmedelsverket. Livsmedelsverkets livsmedelsdatabas version 2015-05-28. 2015 [cited 201511 november]. Available from: http://www7.slv.se/ SokNaringsinnehall/

[30] Narisawa T, Magadia NE, Weisburger JH, et al. Promoting effect of bile acids on colon carcinogenesis after intrarectal instillation of N-methyl-N'-nitro-N-nitrosoguanidine in rats. J Natl Cancer Inst. 1974;53(4):1093-1097.

[31] Chomchai C, Bhadrachari N, Nigro ND. The effect of bile on the induction of experimental intestinal tumors in rats. Dis Colon Rectum. 1974;17(3):310-312.

[32] Gu?Raud F, Tach? S, Steghens J-P, et al. Dietary polyunsaturated fatty acids and heme iron induce oxidative stress biomarkers and a cancer promoting environment in the colon of rats. Free Radic Biol Med. 2015;83:192-200.

[33] Knize MG, Salmon CP, Pais P, et al. Food heating and the formation of heterocyclic aromatic amine and polycyclic aromatic hydrocarbon mutagens/carcinogens. Adv Exp Med Biol. 1999;459:179-193.

[34] Wakabayashi K, Nagao M, Esumi H, et al. Food-derived mutagens and carcinogens. Cancer Res. 1992;52 (7):2092s-8s.

[35] NNR. Sodium as salt, in Nordic nutrition recommendations 2012. In: ministers Nco, editor. Vol. 2014, 515534. Copenhagen: Nordic Council of Ministers; 2012.

[36] Kune GA, Kune S, Watson LF. Dietary sodium and potassium intake and colorectal cancer risk. Nutr Cancer. 1989;12(4):351-359.

[37] Song M, Nishihara R, Wu K, et al. Marine $\omega-3$ polyunsaturated fatty acids and risk for colorectal cancer according to microsatellite instability. JNCI: J Natl Cancer Inst. 2015;107:4.

[38] Calder PC. Omega-3 polyunsaturated fatty acids and inflammatory processes: nutrition or pharmacology? $\mathrm{Br}$ J Clin Pharmacol. 2013;75(3):645-662.

[39] Baena R, Salinas P. Diet and colorectal cancer. Maturitas. 2015;80(3):258-264.

[40] Riondino S. Obesity and colorectal cancer: role of adipokines in tumor initiation and progression. World J Gastroenterol. 2014;20(18):5177-5190.

[41] Aune D, Ursin G, Veierød MB. Meat consumption and the risk of type 2 diabetes: a systematic review and meta-analysis of cohort studies. Diabetologia. 2009;52 (11):2277-2287.

[42] Pan A, Sun Q, Bernstein AM, et al. Red meat consumption and risk of type 2 diabetes: 3 cohorts of US adults and an updated meta-analysis. Am J Clin Nutr. 2011;94 (4):1088-1096. 